\title{
The Synthesis of a Highly Reactive Heterocyclic Reactant and its Unusual Photochemistry; Mechanistic and Exploratory Organic Photochemistry ${ }^{1,2}$
}

Howard E. Zimmerman* and Alexei Pushechnikov

Contribution from the Department of Chemistry, University of Wisconsin-Madison,

Madison, Wisconsin 53706

Howard E. Zimmerman* and Alexei Pushechnikov

\section{Supporting Information}

\section{Mannich condensation of Diphenylacetaldehyde with Paraformaldehyde and Methylamine hydrochloride.}

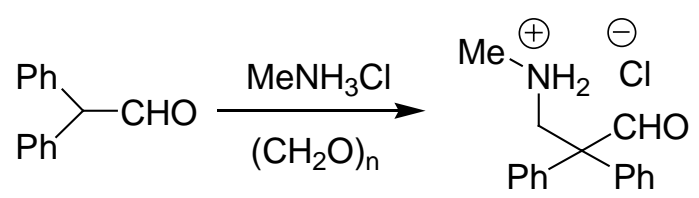

Diphenylacetaldehyde (10 g, $0.0516 \mathrm{~mol})$, paraformaldehyde $(1.4 \mathrm{~g}, 0.048 \mathrm{~mol})$ and methylamine hydrochloride ( $2.5 \mathrm{~g}, 0.037 \mathrm{~mol})$ were mixed and the mixture was heated on a steam-water bath shaking periodically until homogenized. Then the reaction mixture was heated on a steam-water bath at $98{ }^{\circ} \mathrm{C}$ for $4 \mathrm{~h}$, and finally cooled to room temperature affording a semi-solid. The crude product was triturated with ether and filtered to yield $7.0 \mathrm{~g}$ (70\% based on methylamine hydrochloride) of amorphous and very hygroscopic creamy powder, used directly in the next step. ${ }^{1} \mathrm{H}$ NMR $(300 \mathrm{MHz}$, $\left.\mathrm{CDCl}_{3}, \mathrm{ppm}\right): \delta 2.32(3 \mathrm{H}, \mathrm{s}), 3.87(2 \mathrm{H}, \mathrm{s}), 7.13-7.39(10 \mathrm{H}, \mathrm{m}), 7.98(1 \mathrm{H}, \mathrm{s}), 8.93(2 \mathrm{H}$, broad).

Reaction of 3-(Methylamino)-2,2-diphenylpropionaldehyde Hydrochloride with Potassium Cyanate.

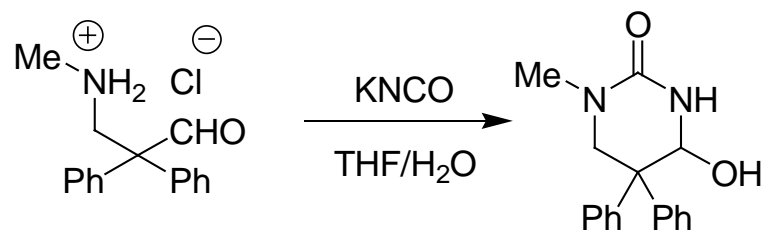


To a stirred solution of $7.0 \mathrm{~g}$ of 3-(methylamino)-2,2-diphenylpropionaldehyde hydrochloride $\quad(0.026$ mol) dissolved in a THF-water mixture $(50 \mathrm{ml}, 1: 1)$ was added $3.1 \mathrm{~g}(0.038 \mathrm{~mol})$ of potassium cyanate, and the reaction mixture was stirred at room temperature for $48 \mathrm{~h}$. The solution was then diluted with $25 \mathrm{ml}$ of water and extracted twice with dichloromethane $(30 \mathrm{ml}$ and $15 \mathrm{ml})$. The organic fractions were combined, dried and concentrated in vacuo. A viscous residue was dissolved in benzene $(30 \mathrm{ml})$ and the solution was refluxed for $5 \mathrm{~min}$. After cooling to room temperature the product crystallized. Recrystallization from benzene yielded $4.6 \mathrm{~g}$ (64\%), mp 193-196 ${ }^{\circ} \mathrm{C}$ decomp. ${ }^{1} \mathrm{H}$ NMR (300 MHz, $\left.\mathrm{CDCl}_{3}, \mathrm{ppm}\right): \delta 2.89(3 \mathrm{H}, \mathrm{s}), 3.77(1 \mathrm{H}, \mathrm{d}, J=12 \mathrm{~Hz}), 4.21(1 \mathrm{H}, \mathrm{d}, J=12 \mathrm{~Hz}), 5.1(1 \mathrm{H}, \mathrm{d}, J=3.6 \mathrm{~Hz})$, $5.48(1 \mathrm{H}, \mathrm{t}), 6.38(1 \mathrm{H}, \mathrm{d}, J=4.5 \mathrm{~Hz}), 7.13-7.27(10 \mathrm{H}, \mathrm{m}) .{ }^{13} \mathrm{C} \mathrm{NMR}\left(75 \mathrm{MHz}, \mathrm{CDCl}_{3}, \mathrm{ppm}\right): \delta 35.0$ $\left(\mathrm{CH}_{3} \mathrm{~N}\right), 48.7\left(\mathrm{CPh}_{2}\right), 51.1\left(\mathrm{CH}_{2}\right), 77.9(\mathrm{NCHO}), 126.6,126.7,128.8,127.3,128.5,128.6(\mathrm{Ph} \mathrm{CH}$ 's $)$, 142.8, $143.1(\mathrm{Ph}, \mathrm{C}$ 's), $155.4(\mathrm{C}=\mathrm{O})$ (shift correlation was confirmed by DEPT-135). HRMS (ESI): $\left[\mathrm{C}_{17} \mathrm{H}_{18} \mathrm{~N}_{2} \mathrm{O}_{2}+\mathrm{Na}\right]^{+}$calcd.: 305.1266 , found - 305.1261.

\section{Sulfone Formation from Tetrahydro-4-hydroxy-1-methyl-5,5-diphenylpyrimidin- 2(1H)-one.}

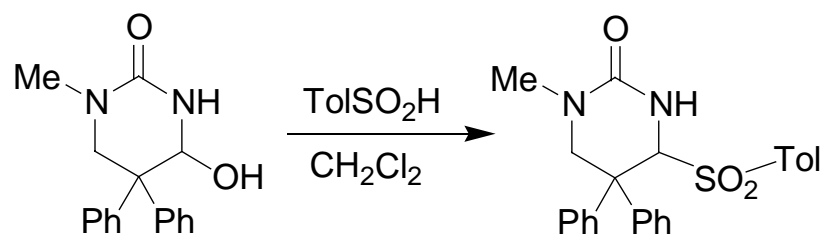

To a cooled $\left(-28{ }^{\circ} \mathrm{C}\right)$ solution of tetrahydro-4-hydroxy-1-methyl-5,5-diphenylpyrimidin-2(1H)one $(3.0 \mathrm{~g}, 0.0106 \mathrm{~mol})$ in $100 \mathrm{ml}$ of dry dichloromethane, p-toluenesulfinic acid $(2.5 \mathrm{~g}, 0.016 \mathrm{~mol})$ and dry sodium sulfate (ca. $2 \mathrm{~g}$ ) were added. The mixture was shaken until a clear solution was formed (ca. $2 \mathrm{~min}$ ) and kept at $-28{ }^{\circ} \mathrm{C}$ for $20 \mathrm{~min}$ and additionally for $2 \mathrm{~h}$ at $0-5{ }^{\circ} \mathrm{C}$. The resulting solution was filtered to remove sodium sulfate. Dichloromethane was removed in vacuo at room temperature and the residue was crystallized with benzene. The crude product was recrystallized from etherdichloromethane (2:1). to yield $3.6 \mathrm{~g}(81 \%)$, mp 179-181 ${ }^{\circ} \mathrm{C}$ decomp. ${ }^{1} \mathrm{H}$ NMR (300 $\mathrm{MHz}, \mathrm{CDCl}_{3}$, ppm): $\delta 2.3(3 \mathrm{H}, \mathrm{s}), 2.8(3 \mathrm{H}, \mathrm{s}), 3.68(1 \mathrm{H}, \mathrm{dd}, J=11.7 \mathrm{~Hz}, J=1.8 \mathrm{~Hz}), 5.16(1 \mathrm{H}, \mathrm{d}, J=11.7 \mathrm{~Hz}), 5.3$ $(1 \mathrm{H}, \mathrm{d}, J=3.3 \mathrm{~Hz}), 6.37(1 \mathrm{H}, \mathrm{d}, J=3.9 \mathrm{~Hz}), 7.06-7.26(12 \mathrm{H}, \mathrm{m}), 7.42(2 \mathrm{H}, \mathrm{d}, J=9.9 \mathrm{~Hz}) .{ }^{13} \mathrm{C} \mathrm{NMR}$ (75 MHz, $\mathrm{CDCl}_{3}$, ppm): $\delta 21.0,35.6,46.8,52.5,76.6,126.1,127.1,127.6,128.1,128.2,128.8,128.9$, 129.7, 135.1, 144.4, 153.4. Anal. calcd. for $\mathrm{C}_{24} \mathrm{H}_{24} \mathrm{~N}_{2} \mathrm{O}_{3} \mathrm{~S}$ : C, 68.55; H, 5.75; N, 6.66. Found: C, 68.44; H, 5.92; N, 6.56. 


\section{5,6-Dihydro-1-methyl-5,5-diphenylpyrimidin-2(1H)-one by Sulfone Elimination.}

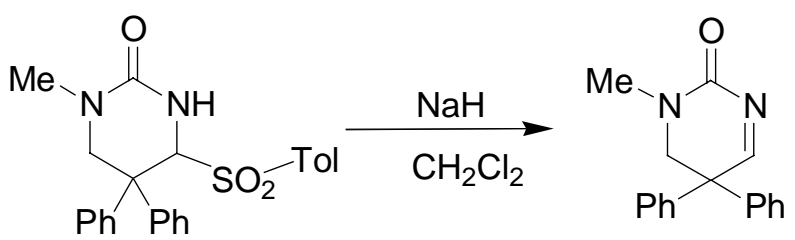

To a stirred suspension of $\mathrm{NaH}(0.12 \mathrm{~g}, 4.76 \mathrm{mmol}, \sim 0.2 \mathrm{~g}$ of $60 \%$ dispersion in mineral oil, washed with dry pentane) in $50 \mathrm{ml}$ of cooled $\left(\sim 0{ }^{\circ} \mathrm{C}\right)$ dry dichloromethane, $1.0 \mathrm{~g}(2.38 \mathrm{mmol})$ of powdered sulfone was added. All manipulations were performed under an atmosphere of dry nitrogen. The reaction was stirred at $\sim 0{ }^{\circ} \mathrm{C}$ for $2 \mathrm{~h}$, and then at room temperature an additional $14 \mathrm{~h}$. Then the reaction mixture was filtered under an atmosphere of dry nitrogen. The filtrate was concentrated in vacuo to dryness. The residue was washed with dry pentane under an atmosphere of dry nitrogen and solvent was decanted. The crude product was recrystallized from dry benzene $(10 \mathrm{ml})$. The yield was $0.42 \mathrm{~g}(67 \%), \mathrm{mp} 209.5-210.5{ }^{\circ} \mathrm{C}$ (sealed tube). ${ }^{1} \mathrm{H}$ NMR $\left(300 \mathrm{MHz}, \mathrm{CDCl}_{3}, \mathrm{ppm}\right): \delta 3.07(3 \mathrm{H}, \mathrm{s})$, $3.92(2 \mathrm{H}, \mathrm{s}), 7.12-7.19(6 \mathrm{H}, \mathrm{m}), 7.33-7.42(6 \mathrm{H}, \mathrm{m}), 8.82(1 \mathrm{H}, \mathrm{s}) .{ }^{13} \mathrm{C} \mathrm{NMR}\left(75 \mathrm{MHz}, \mathrm{CDCl}_{3}, \mathrm{ppm}\right): \delta$ $35.8,50.2,56.0,126.4,127.3,127.8,129.0,140.3,155.6,177.4$.

\section{Photolysis of 5,6-Dihydro-1-methyl-5,5-diphenylpyrimidin-2(1H)-one.}

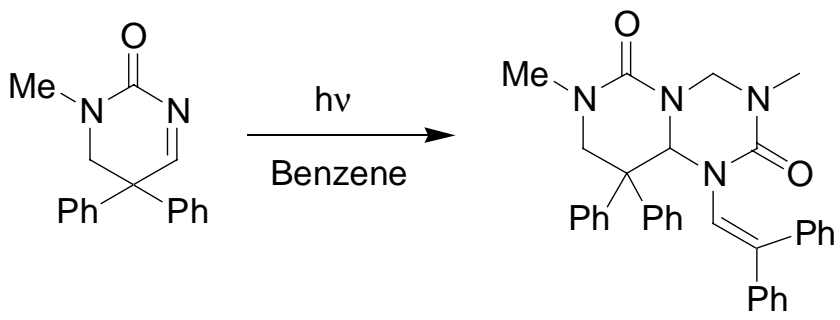

The starting pyrimidinone reactant $(0.40 \mathrm{~g}, 1.52 \mathrm{mmol})$, dissolved in dry benzene $(50 \mathrm{ml})$ was degassed in vacuo for $2 \mathrm{~min}$ in a $250 \mathrm{ml}$ flask filled with dry oxygen-free nitrogen. The procedure was repeated and the solution was transferred to a vacuum-dried $50 \mathrm{ml}$ immersion apparatus for photolysis under an atmosphere of dry oxygen-free nitrogen. The photolysis was performed in the irradiation well with a $100 \mathrm{~W}$ medium pressure mercury lamp with circulated $0.20 \mathrm{M}$ water solution of $\mathrm{CuSO}_{4}$ (wavelength 310-vis) as an UV-filter. After $10 \mathrm{~h}$ of irradiation the reaction mixture was filtered to remove the oxy-dimer $(0.11 \mathrm{~g}, 26 \%)$, and the filtrate was concentrated in vacuo. The residue was separated by column chromatography (silica gel mesh 60-200, ethyl acetate). The last fraction contained the major photolysis product $(0.086 \mathrm{~g}, 21 \%)$. Additional elution with a methanol- 
dichloromethane (1:9) mixture provided the hydroxy-product (tetrahydro-4-hydroxy-1-methyl-5,5diphenylpyrimidin-2(1H)-one) (0.102 g, 24\%) and the oxy-dimer (0.041 g, 10\%). Overall mass balance $81 \%$. The new compound was recrystallized from hexane-ether, mp 212-213 ${ }^{\circ} \mathrm{C} .{ }^{1} \mathrm{H}$ NMR $(300 \mathrm{MHz}$, $\left.\mathrm{CDCl}_{3}, \mathrm{ppm}\right): \delta 2.44(3 \mathrm{H}, \mathrm{s}), 2.94(3 \mathrm{H}, \mathrm{s}), 3.54(2 \mathrm{H}, \mathrm{AB}, J=17 \mathrm{~Hz}), 3.79(1 \mathrm{H}, \mathrm{d}, J=11.4 \mathrm{~Hz}), 4.78$ $(1 \mathrm{H}, \mathrm{d}, J=11.4 \mathrm{~Hz}), 5.58(1 \mathrm{H}, \mathrm{s}), 6.35(1 \mathrm{H}, \mathrm{s}), 6.59(1 \mathrm{H}, \mathrm{s}), 6.6(1 \mathrm{H}, \mathrm{s}), 7.09-7.49(20 \mathrm{H}, \mathrm{m}) .{ }^{13} \mathrm{C}$ NMR (75 MHz, $\left.\mathrm{CDCl}_{3}, \mathrm{ppm}\right): \delta 32.7,34.8,52.1,56.5,57.6,74.6,126.1,127.4,127.9,127.9,128.5,128.7$, $128.7,128.9,129.5,129.7,137.4,138.2,139.3,139.4,142.0,153.2,157.9$. HRMS (ESI): $\left[\mathrm{C}_{34} \mathrm{H}_{32} \mathrm{~N}_{4} \mathrm{O}_{2}+\mathrm{Na}\right]^{+}$calcd. - 551.2423, found - 551.2414.

\section{Oxy-Dimer (5)}

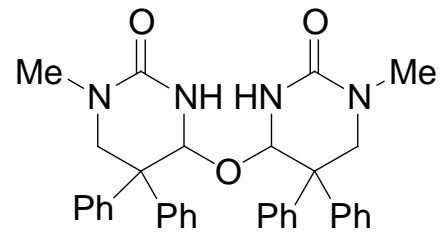

This compound was observed along with the hydroxy hterocycle, tetrahydro-4-hydroxy-1-methyl-5,5diphenylpyrimidin-2(1H)-one, in the photolysis of 5,6-dihydro-1-methyl-5,5-diphenylpyrimidin-2(1H)one as well as in the synthesis described above. The compound is a white microcrystalline material, $\mathrm{mp}$ 226-228 ${ }^{\circ} \mathrm{C}$ decomp. ${ }^{1} \mathrm{H}$ NMR $\left(300 \mathrm{MHz}, \mathrm{CDCl}_{3}, \mathrm{ppm}\right): \delta 2.81(6 \mathrm{H}, \mathrm{s}), 3.45(2 \mathrm{H}, \mathrm{d}, J=11.4 \mathrm{~Hz}), 3.59$ $(2 \mathrm{H}, \mathrm{d}, J=11.4 \mathrm{~Hz}), 5.71(1 \mathrm{H}, \mathrm{d}, J=4.8 \mathrm{~Hz}), 6.93-7.21(20 \mathrm{H}, \mathrm{m}), 7.37(2 \mathrm{H}, \mathrm{m})$. HRMS (ESI): $\left[\mathrm{C}_{34} \mathrm{H}_{34} \mathrm{~N}_{4} \mathrm{O}_{3}+\mathrm{Na}\right]^{+}$calcd. - 569.2529, found - 569.2540.

The CIF File - A Zim034.cif is available from the Cambridge Data Base. 